\title{
The Neutron as an Alternative Explanation for the Trans GZK Cosmic Rays
}

\author{
Adolfo De Unánue, Sarira Sahu, and Daniel Sudarsky \\ Instituto de Ciencias Nucleares, \\ Universidad Nacional Autonoma de Mexico, \\ Circuito Exterior C.U., A. Postal 70-543, 04510 Mexico D.F.
}

\begin{abstract}
We consider the possibility that the Ultra High Energy Cosmic Rays arriving to Earth might be neutrons instead of protons. We stress that in such case the argument for the GZK cutoff is weaker and that it is conceivable that neutrons would not be affected by it. This scenario would require the neutron to start with an energy larger than the observed one, in order to be able to travel the distances involved, within its proper life-time. It must then loose most of the extra energy through interaction with the galactic dark matter or some other matter in the intergalactic medium.
\end{abstract}




\section{Introduction}

Cosmic rays have often been the source of great insights into the workings of the cosmos and the fundamental laws of physics. The most energetic of these cosmic rays are thought to be ultra high energy protons, originating at distances of more than $100 \mathrm{Mpc}$. The distribution of the particle showers, which are probably hadronic, has not indicated so far any appreciable anisotropy, although the statistics for these high energy events is rather poor so far. Fortunately, the Auger project is expected to clarify the situation soon (its initial results do not seem to offer a conclusive answer ${ }^{1}$ ). There is however an almost inescapable expectation that there would be a sharp cutoff in the energies of these showers, as the highly energetic primary protons would interact with the CMB photons loosing most of their energies, until they drop below $\sim 3 \times 10^{19} \mathrm{eV}$, the so called GZK cutoff ${ }^{2,3}$. This conclusion relies essentially on the measured proton energy loss and cross section for the $p+\gamma_{C M B} \rightarrow \Delta^{0} \rightarrow p+q_{\pi} \pi$ processes, the well established thermal distribution of the CMB photons with $T \approx 2.7 K^{o}$ and the Lorentz invariance which allows us to connect the rest frame of the UHECR with the laboratory frame where measurements of the above mentioned cross sections are carried out. The observation during the last years of a series of events with energies quite above the GZK barrier, has produced a lot of excitement in various communities. First and foremost, in the community of cosmic ray physicists, but also in a sector of the community working on tests of fundamental symmetries and/or in quantum gravity. In fact the last few years have witnessed the resurgence of interest into the possible existence of a violation of strict Lorentz invariance associated with the microstructure of the space-time fabric, a type of ether-like feature in the Universe, presumably connected to the quantum mechanical aspects of gravitation. As it turns out the above mentioned absence of the GZK cutoff has played a central role as a motivation for much of the ongoing excitement about the possibility of violation or modification of exact Lorentz Symmetry ${ }^{4,5,6,7,8}$. These proposals face severe problems. In the first case, where one assumes that Lorentz Invarance is broken by the existence of a preferential rest frame tied somehow to the nature of space time associated with Quantum Gravity, the problem resides in the fact that although one might initially postulate that the effects would be only noticeable at very high energies, the appearance of arbitrarily high energy particles in the radiative corrections to any physical process, would transfer the influence of the privileged frame to low energy processes where the effects would have been observed long ago ${ }^{9}$. In the other schemes where one hopes to deform the representation 
of the Lorentz algebra by some nonlinear terms, the problem is that one ends with an scheme in which the addition of our momenta depends of the ordering of the particles, the existence of high energy particles even in remote regions of the universe would affect the local processes, or one would rule out bodies with masses larger than the Planck mass. All these problems are of course so serious that one can not hope (despite the efforts of some of its proponents) to use these schemes to address any phenomenological issue, at least until those problems are resolved. For a more extensive discussion of these issues see Ref.[10, 11, 12, 13, 14, 15]. Furthermore, in view of the radical nature of any proposal challenging the validity of Special Relativity, and the serious problems encountered by the existing proposals it seems worthwhile to search for less exotic alternatives to understand the apparent absence of GZK cutoff. It is clear that all proposals to address the problem at hand will be "exotic" to some degree ( otherwise there would not be a problem), thus we must emphasize that the criteria of exoticity in this context must be taken as a relative one. This relative scale is in this case very high and is represented by the proposals that challenge the validity of Special Relativity, which as we have indicated face enormous (perhaps insurmountable) obstacles.

The observation of such ultra high energy cosmic rays poses, in fact, two challenges: What is exactly the mechanism that accelerates them to those energies? and, how do they manage to travel beyond $50 \mathrm{Mpc}$ despite the expectation of a GZK cutoff? The first issue represents a challenge mainly to astrophysics, because the Hilles criteria ${ }^{16}$ indicates that the simplest acceleration mechanisms could not produce particles with the desired energies in systems that are known to be within a reasonable distace. The second issue represents a very serious challenge to fundamental physics as even if one could find a mechanism to produce the highly energetic particles, the well established low energy physics of proton photon interaction together with the ubiquitous cosmic microwave photon distribution should prevent such particles from traveling the required distances without loosing most of their energy. The critical nature of this challenge can be noted by the apparent willingness of the physics community to consider doing away with so cherished, basic and well established principles as those underlying Spatial Relativity. This article will thus mainly address the second issue while the first one, regarded as essentially an astrophysical puzzle to be tackled independently, will only be touch upon in order to describe some alternatives that have been considered so far.

There have been of course many proposals to deal with this problem, all of them involving new physics in some way or another. The fact that the observed energies of 
the UHECRs pose also a serious challenge to the acceleration mechanism in conventional astrophysical objects, explains the enhanced atractiveness of proposals that involve new energy sources for the production of UHECRs ${ }^{17}$. For instance, it has been suggested that, the UHECRs can be produced in the decay of very massive $\left(m_{X} \geq 10^{12} \mathrm{GeV}\right)$ and long lived X particles, originating from high-energy processes in the early Universe ${ }^{17,18}$, the so called "top down model". However, these particles must decay within one GZK interaction length to avoid energy loss. At the same time, their density must be large enough to give detectable flux of UHECRs. A potential problem with this scenario is that, it predicts higher flux of UHE photons than protons. Another recent approach proposes that a strong galactic halo sized magnetic field as a mechanism for isotropization of the flux of UHECR of nearby origin ${ }^{19}$, but unfortunately this scenario poses serious astrophysical problems ${ }^{20}$.

We will focus here on the possibility that the ultra high energy cosmic rays are neutrons instead of protons. There are several reasons for doing this. First, the neutron is almost identical to the proton as far as its hadronic properties and, thus, the particle showers produced by it would be very similar to those produced by a proton. Second, one of the possible sources of UHECR are compact object mergers, and these objects have neutrons as their major constituent. Third, despite what might appear at first sight, essentially all astrophysical mechanisms that produce high energy protons, are equally good sources for high energy neutrons; the reason is, of course, the inverse beta decay of the energetic protons in interaction with the electrons in the astrophysical source. The problem is of course the mechanism of acceleration resulting in such highly energetic particles. As we have said our point of view here will be to address the fundamental physics issues and regard this last but important issue to be an essentially astrophysical problem to be touched upon only superficially. Finally, there is almost no direct experimental data of the way neutrons interact with photons (or other particles) in the energy regime of interest $^{21,22}$, and thus, any suggestion of a conflict with special relativity would be based on theoretical arguments which could in turn be subject to questioning. In fact, the neutral character of the neutron suggests, a priori, that its interaction with the photons of the cosmic microwave background would be substantially reduced as compared with that of the proton. On the other hand, one could estimate the energy loss and cross section for the $n+\gamma_{C M B} \rightarrow \Delta^{0} \rightarrow n+q_{\pi} \pi$ process, and get a neutron mean free path similar to that of the proton. However, we should keep in mind that this analysis does not rest on unshakable grounds. In fact, the strongest theoretical arguments on which these sort of calculation relies on, are the phenomenological models known as Vector Meson Dominance 
(VMD) or its variants such as Photon Hadronic Structure Approaches (PHSA), which are themselves not Lorentz covariant ${ }^{23}$.Thus, it seems a logical possibility, that in the search for the "relatively least exotic options", one should question such theoretical predictions if they are not corroborated by independent empirical evidence. The point is in fact, not only that questioning the Lorentz Violating VMD seems far less radical than questioning Special Relativity, but also that it would be logically inconsistent to question Special Relativity, based, in part, on the use of a Lorentz Violating postulate.

We emphasize that we do not challenge, in principle, the standard model as the correct theory to treat the neutron's interaction with other known particles. However, at the energies of interest QCD, is not necessarily in its perturbative regime and the procedures to do calculations are not fully reliable. In other words, we do not have a fully proven algorithm to calculate the neutron cross-sections with different particles at these energies. We must recall that the crux of the argument for the GZK cutoff for protons relies in the well established pion photo-production cross section at the center of mass energy of the $\Delta$ resonance, a reaction in which the high energy proton looses an important part of its energy ( of order $20 \%$ per reaction). Above this threshold the cross section decreases rapidly by a factor of 7 . This is the regime where we question the identification of the measured characteristics of the proton-photon interactions with those corresponding to photon-neutron. At higher neutron energies $E$ the center of mass energy $s$ becomes larger (recall that $s$ grows like $E^{1 / 2}$ ), eventually reaching the point where perturbative QCD calculation should be reliable, and the neutron and proton cross section and energy losses via pion photo production or other channels should become nearly identical ( except for a factor $2 / 3$ corresponding due to the difference in the sum quarks charges squares). This should happen when the center of mass energy for the photon- quark ( or photonparton) system reaches the several $100 \mathrm{MeV}$ level, corresponding to a neutron energy of about $E \approx 10^{21} \mathrm{eV}$ depending on the exact percentage of the neutron energy carried by the corresponding parton. However then the cross section should be essentially $2 / 3$ of the corresponding proton cross section which is a factor of about 7 smaller than at the $\Delta$ resonance $^{24}$ (so between the two effects one should get an full order of magnitude decrease in the cross section), while the mean energy loss per reaction remain very similar and certainly do not change enough to compensate for the above factors ${ }^{25}$. Thus it is unclear whether this would be a problem for the model.

In order to make a reasonable lower bound estimate of the energy loss of neutrons with the CMB we will use only the phenomenological Pauli interaction characterizing 
the nucleon's magnetic moment coupling to photons. On the other hand, regarding the nucleon interaction with the exotic particles, the situation is a total unknown therefore we will treat this part at a purely phenomenological level.

The obvious problem that our proposal would encounter is that even at energies like $10^{20} \mathrm{eV}$ a neutron would, due to its finite lifetime, manage to transverse only a fraction of the $100 \mathrm{Mpc}$ that separate us from the likely sources of these energetic particles. The obvious solution is to assume that the neutron is in fact substantially more energetic, thus allowing it to cover the distance involved, and, that it looses most of its energy, due to interactions with matter during its trip toward us, so that it arrives to Earth with the $10^{20} \mathrm{eV}$ of the observed showers.

We will be considering in fact two scenarios: In the first, the neutron looses its "excess" energy very slowly during its whole intergalactic trip, through its interaction with some unknown component of the intergalactic media. In the second scenario the neutron travels through the intergalactic media, interacting only with the CMB of photons and neutrinos, and loosing almost no energy, while at the end of the trip it losses its "excess" energy interacting with the dark matter in the galactic halo.

\section{General Requirements}

The requirements on the models are then twofold: That the neutron travels from a source at $D_{S}=10^{2} \mathrm{Mpc}$ and arrives to Earth with an energy of $10^{20} \mathrm{eV}$, and, that the matter responsible for the energy loss should not have been already detected. The natural candidate for this matter would be the dark matter in our galactic halo, or the cosmological dark matter.

The first part is achieved by assuming that the neutron has an initial energy of at least $10^{22} \mathrm{eV}$ so that during its mean lifetime of $\tau_{L}=10^{3}$ sec it would travel a distance of $c \gamma 10^{3} \mathrm{sec}=100 \mathrm{Mpc}$. There are two issues here, the identity of the particles and their energy source. Regarding the first point we have already noted that once protons are produced in any electron rich medium, neutrons with essentially the same energy can be readily obtained via inverse beta decay. In fact very high energy neutrons might have already been observed ${ }^{26}$ coming from our galactic center, and it has been estimated that particles above the GZK energy coming along the line of sight from CenA (about 3.4 Mpc away), are much more likely to be neutrons than protons ${ }^{27}$. Theoretical estimations also indicate that neutrons should be a nontrivial fraction of the cosmic rays around the GZK 
cutoff for nearby sources ${ }^{28}$. Regarding the source of the very energetic particles, we point out that nobody knows exactly what is the mechanism producing the energetic protons, neutrons or other particles with energies of the order of $10^{20} \mathrm{eV}$. The diffusive shock acceleration mechanism (DSAM), is currently the standard theory of CR acceleration, but when applied to supernova, it can acclerate particles up to $10^{17} \mathrm{eV}$. So, for UHECR beyond $10^{17} \mathrm{eV}$, one has to invoke shocks on larger scale, for example in AGNs and radiogalaxies. In fact, hot spots in Fanaroff-Riley type II radio galaxies ${ }^{29,30,31}$, about $100 \mathrm{Mpc}$ away, and "Dead Quasars" 32 have been estimated to reach the $10^{21} \mathrm{eV}$ mark, also Blazar jets $^{33}$ have been estimated to be potential sources of protons with energies well above the $10^{20} \mathrm{eV}$, while a recent proposal based on the so called "Plasma Wake Field Acceleration Mechanism", seems to be able, in principle to accelerate particles to any energy ${ }^{34}$. As, we noted above, our model requires sources of about $10^{22} \mathrm{eV}$ neutrons, which, given the above examples and the astrophysical uncertainties, can not be excluded. As we have already acknowledged this scheme makes the astrophysical problem posed by the existence of cosmic rays with energies of order $10^{20} \mathrm{eV}$ even more dramatic by requiring particles of energies of order $10^{22} \mathrm{eV}$ but in light of the problematic aspects of other alternatives this seems a relatively small price to pay.

The loss of energy occurs either along the whole distance from the source $D_{S}$ or just on the last section $D_{L}$ corresponding to the traversing of the galactic halo of the Milky Way which is about $600 \mathrm{kpc}$.

Regarding the second requirement we note that the SuperKamiokande detector relies on Cêrenkov radiation. The recoil velocity of a nucleon moving with the Earth due to its interaction with a dark matter particle at rest in the frame of the galaxy would be at most $400 \mathrm{~km} / \mathrm{s}$, and this velocity is too low to lead to Cêrenkov radiation. Another check is that the interaction of celestial bodies -like that of Earth in its motion with the sun around the galactic center, or that of the galaxy itself in the intergalactic medium -with these dark particles should not lead to noticeable effects. In fact a bound for this effect can be easily estimated for any ordinary object of mass $\mu$ and linear size $R_{o}$ as it travels through the dark matter gas with velocity $v$. We envision these dark matter particles as Weakly Interacting Massive Particles (WIMPs) and analyze the issue from the rest frame of the dark matter gas. It is easy to see that the change in energy $E_{o}$ of the body in one interaction with one WIMP of mass $M$ initially at rest is about $\Delta E_{o}=-M v^{2}$. We can evaluate the change per unit time in the object's kinetic energy by simple considerations 
leading to the following estimate for the fractional energy loss

$$
\frac{\Delta E_{o}}{E_{o}} \approx-\frac{\rho_{D M}}{\rho_{o}} \frac{v \Delta t}{R_{0}} \times \operatorname{Min}\left(1, \sigma\left(\rho_{o} / m\right) R_{o}\right)
$$

Now we consider, for instance the energy loss of Earth as it travels through the galactic Halo during the four billion years of the Earth's existence. Using the density of the Dark Matter Halo, $\rho_{D M}=0.3 \mathrm{GeV} / \mathrm{cm}^{3}$ Ref.[35], we find $\frac{\Delta E}{E} \approx 10^{-9} \times \operatorname{Min}\left(1, \sigma\left(\rho_{o} / m\right) R_{o}\right)$. Thus we conclude that these considerations lead to no useful constraint.

Finally we must address the tight bounds on the cross section of nucleon-WIMP interaction for those WIMPs that might traverse our laboratories on Earth. These are obtained by looking at the scintillation of heavy nucleus recoiling from collision with WIMPS that might constitute the galactic dark matter halo. The best such bounds are of the order $10^{-42} \mathrm{~cm}^{2}$ for he nucleus-WIMP cross section. We transform these into bounds on the neutron-WIMP cross section as follows:

$$
\sigma_{\text {Nucleus-WIMP }} \approx A^{2 / 3} \sigma_{\text {neutron-WIMP }}
$$

where $A$ is the baryon number of the nucleus which for instance, for Ge - the element employed in the most sensitive experiments to date ${ }^{36,37}$ - is 122 . Thus we find;

$$
\sigma_{\text {neutron-WIMP }} \leq 10^{-43} \mathrm{~cm}^{2}
$$

We next analyze process of the neutron energy loss in its interaction with the dark matter particles.

\section{Analysis of the Energy Loss}

We foccus on the rate of energy loss of an extremely relativistic neutron with energy $\gamma m$ traveling through a gas of dark matter particles of mass $M \neq 0$ assumed to be essentially at rest in our local comoving frame or that of our galaxy. The neutron energy loss in one collision depends only on its scattering angle $\theta$ and in fact we have: $\left(\gamma \gamma^{\prime}-1\right)+(M / m)(\gamma-$ $\left.\gamma^{\prime}\right)=\gamma \gamma^{\prime} \beta \beta^{\prime} \cos (\theta)$ where $\gamma$ and $\gamma^{\prime}$ are the Lorentz factors for the incoming and outgoing

neutrons while $\beta$ and $\beta^{\prime}$ the corresponding velocities (we are using $c=1$ ). Considering this equation in the extreme cases of $\cos (\theta)= \pm 1$ we find,

$$
\Delta^{(1)} E / E=-2 \frac{(M / m)\left(\gamma-\gamma^{-1}\right)}{\left(1+2 \gamma(M / m)+(M / m)^{2}\right)} .
$$


The condition for energy loss to be smooth is $\Delta \gamma / \gamma<<1$ (i.e., that it might be described as a continuous process). This implies either $M / m<<(2 \gamma)^{-1}$ or $M / m>>2 \gamma$. On the other hand, the requirement of forward scattering requires $M / m<\gamma^{2}$. For the situation at hand, the values of $\gamma$ will be in the range $10^{11}$ to $10^{13}$ so these constraints are satisfied if $10^{13} \mathrm{GeV} \leq M \leq 10^{22} \mathrm{GeV}$. In that case the energy loss in one collision can be estimated as: $\Delta^{(1)} E / E=-2(m / M) \gamma$. Then

$$
\frac{d E}{d t}=\int \Delta^{(1)} E \frac{d N}{d t} \approx \Delta^{(1)} E n_{\text {gas }} \sigma v_{r e l},
$$

where $\frac{d N}{d t}$ is the number of collisions per unit time. The RHS of the last part of the equation has been obtained by assuming that all the collisions have the same energy loss and all the particles in the gas were initially at rest. Using $\Delta^{(1)} E \approx-2 E^{2} / M$ we have $\frac{d E}{d t} \approx\left(-2 E^{2} / M\right)\left(\rho_{D M} / M\right) \sigma$, where we have set $v_{r e l}=1$ and $n_{g a s}=\rho_{D M} / M$.

We carry our analysis assuming that, in general, $d E / d t=-\mathcal{C} E^{n}$. Note that, as the energy of our neutron in the rest frame of the gas of WIMPS is a Lorentz scalar, such form does not imply any breackdown of Spacial Relativity. $\mathcal{C}$ is a constant that depends on the various parameters of the model. We then have, $d E / d \tau=-(E / m) \mathcal{C} E^{n}$. Considering $n$ non-negative we have:

$$
\frac{1}{E_{f}^{n}}-\frac{1}{E_{i}^{n}}=\frac{\mathcal{C}}{m} n \Delta \tau
$$

for $n>0$; or $E_{f}=E_{i} e^{-\frac{c}{m} \Delta \tau}$ for $n=0$. Let us examine first the case $n>0$ : Using $E_{i} \geq 100 E_{f}$, we find, $\mathcal{C} \approx m /\left(n \Delta \tau E_{f}^{n}\right)$. We can express the energy loss in terms of the distance traveled. The infinitesimal distance is given by $d x=\beta d t \simeq \gamma d \tau=(E / m) d \tau$, where we used the fact $\beta \simeq 1$. Therefore, $d E / E^{n}=-\mathcal{C} d x$ and

$$
D=\frac{1}{\mathcal{C}(n-1)}\left(E_{f}^{(1-n)}-E_{i}^{(1-n)}\right) \approx \frac{E_{f}}{m}\left(\frac{n}{n-1}\right) \Delta \tau .
$$

While for the case $n=1$ we find, $E_{f} / E_{i}=e^{-\mathcal{C D}}$. Armed with these results, we now proceed to a more detailed analysis of the two different scenarios.

\section{Study of Different Scenarios}

Consider first the scenario where the neutron's energy loss occurs along the whole trip from the source to the Earth. Here we need $D>100 \mathrm{Mpc}$ and $\Delta \tau<\tau_{L}=10^{3}$ sec, while $E_{f}=10^{11} \mathrm{GeV}$ and $m=1 \mathrm{GeV}$. Thus for $n>0$, using Eq.(7), we obtain $n /(n-1)>$ 
$10^{2}$. So we need to be essentially in the $n=1$ case: Defining $a=\frac{\Delta \tau}{\tau_{L}}$, so $a<1$, and combining $E_{f} / E_{i}=e^{-\mathcal{C D}}$, with Eq.(6) we find $\left(m / E_{f}\right)\left(1-e^{-\mathcal{C D}}\right)=\mathcal{C} a \tau_{L}$. Thus defining $x=\mathcal{C D}$ and using the known values for $E_{f}, m, \tau_{L}$ and $D$ we find $1-e^{-x}=10^{-2} a x$. For $a \sim 1$ we have $x \approx 10^{2} / a$. We will have $n=1$ if the cross section is essentially $\sigma=y E^{-1}$ with $y$ some constant. Thus $\mathcal{C}=2 y \rho_{D M} M^{-2}$. For the dark matter density in the intergalactic medium we take the value $\rho_{D M}=\alpha \rho_{C r i t}$ with $\alpha \approx 0.3$ as indicated by the latest cosmological data ${ }^{38}$. We use $\rho_{\text {Crit }}=h^{2} \times 10^{-5} \mathrm{GeV} / \mathrm{cm}^{3}$ with $h$ the standard characterization of the Hubble parameter. We thus obtain the expression for the cross section $\sigma=\left(2 a h^{2}\right)^{-1}\left[M^{2} /\left(E \times 10^{13} \mathrm{GeV}\right)\right] \times 10^{-6} \mathrm{~cm}^{2}$. This model requires then a cross section that at low energies is extremely large. This would seem to rule out this particular scenario. This conclusion might be avoided if for some reason the type of WIMPs that make up the intergalactic matter are not present within the galaxy. After all our galaxy is located within a halo made out of of Dark Matter, which could be of a very different nature from the intergalactic dark matter (in fact one of them seems to clump very effectively while the other does not) and is conceivable that the two different kinds might repel each other. One more option to avoid dismissing this scenario emerges when we note that there is no need to assume that the particular behaviour that is needed at the energies prevalent in the cosmic ray neutron interaction with the WIMPs extrapolates all the way to the very low energy regimes where the cross section would become huge. One could view the $1 / E$ behaviour of the cross section in the high energy regime as the tail of some resonance, and the low energy regime relevant for the Laboratory dark matter searches, as representing the other side of the tail or more generally assume two very different energy dependences separated by some intermediate threshold. None of these possibilities seems to be very elegant or economic, but we must remain open to such possibilities in part by recalling that if the standard matter sector of particle physics exhibits such richness, the dark sector might do likewise.

In the second scenario where the neutron does not loose any of its energy until it reaches the galactic halo, we assume the cross section behaves as $\sigma=z E^{N}$. Then Eq.(5) becomes

$$
\frac{d E}{d t} \approx-2\left(\rho_{D M} / M^{2}\right) z E^{N+2}
$$

so $n=N+2$ and $\mathcal{C}=2\left(\rho_{D M} / M^{2}\right) z$. Now the condition on the distance traveled becomes $600 \mathrm{kpc}=10^{14} \sec \times\left(\Delta \tau / \tau_{L}\right)(n / n-1)$ which leads to $\left(\Delta \tau / \tau_{L}\right)(N+2 / N+1)=0.6$ which is comfortably compatible with $N \sim 1$ as is clear that the proper time spent in crossing 
the galactic halo $\Delta \tau$ can not be but a fraction of the neutron lifetime $\tau_{L}$. The expression for the constant is thus $\mathcal{C}=1.6 \times 10^{-3} \mathrm{sec}^{-1} m(n-1)^{-1} E_{f}^{-n}$. Setting $\rho_{D M}=0.3 \mathrm{GeV} / \mathrm{cm}^{3}$ the value for the dark matter Galactic halo, we obtain $z=8 \times 10^{-14} \mathrm{~cm}^{2} \frac{1}{N+1}\left(M / E_{f}\right)^{2} E_{f}^{-N}$ and thus

$$
\sigma=8 \times 10^{-14} \mathrm{~cm}^{2}(N+1)^{-1}\left(M / E_{f}\right)^{2}\left(E / E_{f}\right)^{N} .
$$

In order to compare this with the bound of Eq.(3) we must note that the quantity $E$ in the above formulae refers to the energy of the neutron in the rest frame of the WIMP. For the case of the conditions in the laboratory which lead to the bounds in question this energy is that of a $300 \mathrm{~km} / \mathrm{sec}$ neutron interacting with a WIMP, which is essentially the neutron rest energy, thus:

$$
\sigma=8 \times 10^{-14} \mathrm{~cm}^{2}(N+1)^{-1}\left(M / E_{f}\right)^{2}\left(\mathrm{~m} / E_{f}\right)^{N}=\frac{8}{(N+1)} 10^{-10-11 N} \mathrm{~cm}^{2}<10^{-43} \mathrm{~cm}^{2}
$$

where we have used $M=10^{13} \mathrm{GeV}, E_{f}=10^{11} \mathrm{GeV}$ and $m=1 \mathrm{GeV}$. It is clear that the bound can be satisfied for $N>3$. Thus for instance, taking $N=4$ we have $\Delta \tau / \tau_{L}=$ $(6 / 5) \times 0.6<1$.

We have questioned the reliability of the estimations of the neutron CMB photons interactions, and thus we will consider as the minimal reasonable estimate of the loss of energy due to this interaction the result of a calculation based only on the interaction of the magnetic moment of the neutron with photons using the phenomenological lagrangian proposed by Pauli: $\mathcal{L}_{P A U L I}=-\frac{\mu_{p}}{2} \bar{\psi} \sigma_{\mu \nu} \psi F^{\mu \nu}$. We are interested in the elastic scattering between the neutron and the photon, which to lowest order gives, $\frac{d E}{d t} \simeq-10^{-106} \mu_{p}^{4} T^{2} E^{4}$. Using $\mu_{p} \simeq 4.8 \times 10^{-7} \mathrm{MeV}^{-1}, T=2.348 \times 10^{-10} \mathrm{MeV}$, we obtain a value for $\mathcal{C} \simeq 4 \times 10^{-145}$ $\mathrm{GeV}^{-2}$. The energy loss for such neutron, while traversing $100 \mathrm{Mpc}$ is obtained from: $3 D \mathcal{C}=\left(E_{f}^{(-3)}-E_{i}^{(-3)}\right)=1.8 \times 10^{-105} \mathrm{GeV}^{-3}$. Thus, for $E_{f}=10^{11} \mathrm{GeV}$, and assuming this to be the only interaction of neutrons and the CMB photons, the energy loss is negligible on the scales and regimes we are interested on. Of course it is reasonable to expect this to be only a lower bound and there is plenty of room for an increased cross section for neutron photon interaction but as we argued we can not be absolutely certain of its value based on the theoretical tools at our disposal today and the indirect methods for its estimation which in turn rely on phenomenological calculational schemes whose extension to the case at hand that we are questioning. Again the reader should keep in mind that we do this only in he spirit of questioning, among VMD and SR, the least well established of the two. 


\section{Conclusions}

The GZK anomaly is the only existing evidence presented as an argument for Lorentz Invariance Violation. As mentioned before, this is a very problematic proposal. In fact there is, up to this point, no truly congruent model for the violation of Lorentz symmetry $^{10,11,12,13,14,15}$. In view of the highly problematic nature of the proposals for dealing with the GZK anomaly on the basis of questioning of the Lorentz Invariance of physics, we have considered an alternative that is based on the questioning of less well established principles.

We have briefly explored here a couple of alternative scenarios, which have the advantage of not requiring any new form of matter beyond the non-baryonic dark matter whose existence is required to address cosmological and astrophysical issues. The model does indeed aggravate the requirements on the source of high energy particles beyond the levels required in the context of the observed cosmic rays. We just reiterate that this is already, and independently of this model, a problem for astrophysics. It is clear the need for a thorough investigation of the details of the proposals mentioned at the beginning, and the search for new alternative mechanisms to deal with the astrophysical aspect of problem. However the only real new fundamental physics that this scenario requires is an extremely weak interaction of nucleons (baryons) with Dark Matter. One also must be willing to call into question of phenomenological models of hadron-photon interaction, whose extrapolation to neutrons has so far relied only on very indirect experimental studies. This aspects of our proposal have the added attractive feature of making it suitable for experimental exploration within the context of dark matter searches, and more readily in the experimental studies of neutron-photon interactions and their confrontation with models such as VMD. In fact the nucleon-photon interaction in the energy regime of interest is, according to this analysis the most pressing issue to clarify the prospects of this kind of solution to the GZK puzzle. Our main point is that before considering the breakdown of something so fundamental and well tested as Lorentz Invariance, and given the difficulties that such program has encountered so far ${ }^{10,11,12,13,14,15}$, it seems worthwhile to explore other options even if they involve relatively radical assumptions. In this respect we must keep in mind that the degree of radicallity of such assumptions must be measured relative to that of the proposals to do away with special relativity. In this article we have shown that there is room for "relatively simple" explanations for the GZK enigma, when considering that the UHECR might include neutrons. This seems to be an interesting 
option, that calls for further experimental exploration.

\section{Acknowledgments}

We acknowledge very useful comments from Tsvi Piran. This work was supported in part by CONACYT project 43914-F and DGAPA- UNAM projects IN 108103 and IN 119405.

\section{References}

[1] S. Vorobiov [arxiv: astro-ph/0608576] .

[2] K. Greisen, Phys. Rev. Lett. 16, 748 (1966).

[3] G. T. Zatsepin and V. A. Kuzmin, JETP. Lett. 478 (1966).

[4] L. Gonzalez-Mestres, ArXive: physics/9705031.

[5] L. Gonzalez-Mestres, ArXive: physics/971204.

[6] S. R. Coleman, and S. L. Glashow, ArXive: hep-ph/9808446.

[7] J. R. Ellis, N.E. Mavromatos, and D.V. Nanopoulos, Phys. Rev. D63,124025, (2001).

[8] G. Amelino-Camelia, and T. Piran, Phys. Rev. D64,036005, (2001).

[9] J. Collins, A. Perez, D. Sudarsky, L. Urrutia, and H. Vucetich, Phys. Rev. Lett. 93, 191301, (2004).

[10] R. Schützhold and W. G Unruh, JETP Lett. 78, 431, (2003);

[11] J. Rembieliński and K. A. Smoliński , Bull. Soc. Sci. Lett. Lodz 53:57-63,(2003, [arXiv: hep-th/0207031];

[12] D. Sudarsky Int. Jou. Mod. Phys. D 1, 2069, (2005);

[13] C. Chryssomalakos, E. Okon Int. Jou. Mod. Phys. D 13, 1817,(2004);

[14] D. Grumiller, W. Kummer and D. V. Vassilevich, Ukr. J. Phys. 48, 329, (2003) ;

[15] D. V. Ahluwalia-Khalilova, Class. Quantum Grav. 22,1433, (2005). 
[16] A. M. Hillas Astronomy \& Astrophyiscs 22, 425 (1984).

[17] For reviews see P. Bhattacharjee and G. Sigl, Phys. Rep. 327, 109 (2000).

[18] C. T. Hill, D. N. Schramm and T. P. Walker, Astrophysical J. 454, Phys. Rev. D36, 1007, (1987).

[19] G. R. Farrar, and T. Piran, Phys. Rev. Lett. 84, 3527, (2000).

[20] A. Dar, ArcXive: astro-ph/0006013.

[21] T. Bauer and F. Pipkin, Rev. Mod. Phys., 50, 261, (1978).

[22] F. Wissman, "Quasi-Free Compton Scattering"; Springer Tracts in Modern Physics, (2003).

[23] E. Comay, "Remarks on the proton-hadron interactions", Apeiron (2003).

[24] Particle Data Group, The European Physical Journal 15, 232 (2000).

[25] E. Waxxman and J. Bahcall, Astr. Phys. J. 541,707, (2000).

[26] R. M. Crocker et.al, Astrophysical J. 622, 892 (2005).

[27] L. A. Anchordoqui, H. Goldberg, and T. J. Weiler Phys. Rev. Lett. 87, 081101, (2001).

[28] W. Y. P. Hwang and B. Q. Ma Eur. Phys. J. A 25, 467, (2005).

[29] C. A. Normanet. al. Astrophys. J. 454, 60, (1995).

[30] P. L. Biermann J. Phys, G. Nucl. Part. Phys. 23, 1, (1997).

[31] F. Takahara in "Physics and Astrophysics of Neutrinos" M. Fukuhita \&A. Susuki (Eds.) Spinger Tokyo (1994), 900.

[32] E. Bolt and P. Gosh Mon. Not R. Astron Soc 307 491, (1999).

[33] Claus Grupen, "Astroparticle Physics", ( Springer Verlag, Berlin 2005) pg 168.

[34] P. Chen et. al. Phys. Rev. Lett. 89, 161101, (2002).

[35] Particle Data Group Web Page http//pdg.lbl.gov. 
[36] A. Rubbia, TAUP 2005: Proc. Ninth Int. Conf. on Topics in Astroparticle and Underground Physics.

[37] L. Kaufmann, Diploma Thesis (2005).

[38] S. Dodelson, Modern Cosmology, Academic Press, (2003). 